\title{
Scientific factors for assessing biosimilarity and drug interchangeability of follow-on biologics
}

This article was published in the following Dove Press journal:

Biosimilars

I5 June 2011

Number of times this article has been viewed

\author{
Shein-Chung Chow' \\ Laszlo Endrenyi \\ Peter A Lachenbruch ${ }^{3}$ \\ Lan-Yan Yang' \\ Eric $\mathrm{Chi}^{4}$
}

'Duke University School of Medicine, Durham, NC, USA; ${ }^{2}$ University of Toronto, Toronto, Canada; ${ }^{3}$ Oregon State University, Corvallis, OR, USA;

${ }^{4}$ Amgen, Inc, Thousand Oaks, CA, USA
Correspondence: Lan-Yan Yang Post-Doc, Department of Biostatistics and Bioinformatics, Duke University School of Medicine, 2424 Erwin Road, Hock Suite II 02, Room I 1069, Durham, NC 27705, USA

$\mathrm{Tel}+19196688004$

Fax + I 9196685888

Email lan-yan.yang@duke.edu
Abstract: Biological products are therapeutic agents produced using a living system or organism. In practice, access to these life-saving biological products is limited due to their expensive cost. In the next few years, patents of the early biological products will expire. This provides other biopharmaceutical/biotech companies the opportunity to manufacture follow-on biologics. For the conventional pharmaceuticals of small molecules, regulations and statistical methods for the assessment of bioequivalence for generic approval are well established. However, unlike the conventional drug products, the complexity and heterogeneity of the molecular structure, complicated manufacturing process, different analytical methods, and the possibility of severe immunogenicity reactions make evaluation of equivalence (similarity) between an innovator and its follow-on biologics a great challenge for both the scientific community and regulatory agencies. This article reviews past experiences for the assessment of bioequivalence for conventional drug products. Detailed descriptions of the fundamental differences and assumptions between the chemical generic products and follow-on biologics are given. An overview of current regulatory requirements for assessing biosimilarity of follow-on biologics is provided. Statistical considerations for scientific factors for assessing biosimilarity and drug interchangeability of the follow-on biologics as posted at the recent FDA Public Hearing on Approval Pathway for Biosimilar and Interchangeability Biological Products are discussed. In addition, current statistical issues that are commonly encountered when assessing biosimilarity of follow-on biologics are reviewed.

Keywords: bioequivalence, biosimilarity, drug interchangeability, alternating, switching, replicated design, biosimilarity index

\section{Background}

When an innovative drug product is going off patent, generic companies may file an abbreviated new drug application (ANDA) for approval of the generic copies (with identical active ingredient) of the innovative drug product under the Hatch-Waxman Act. For the approval of generic drug products, the US Food and Drug Administration (FDA) requires that evidence of close similarity in average bioavailability be provided through the conduct of pharmacokinetic (PK) bioequivalence (in terms of rate and extent of drug absorption) studies. The assessment of bioequivalence as a surrogate endpoint for the evaluation of drug safety and efficacy is based on the Fundamental Bioequivalence Assumption that if two drug products are shown to be bioequivalent in average bioavailability, it is assumed that they are therapeutically equivalent and can be used interchangeably.

Unlike drug products with identical active ingredients, the concept for the development of generic versions of biologic products is different because these are made 
of living cells. The generic versions of biologic products are referred to as biosimilars by the European Medicines Agency (EMA), follow-on biologics (FOB) by the FDA, or subsequent entered biologics (SEB) by Health Canada.

Biosimilars are fundamentally different from generic chemical drugs. Important differences include the size and complexity of the active substance and the nature of the manufacturing process. Because biosimilars are not exact copies of their originator products, different criteria for regulatory approval may be required even though the principles of evaluating bioequivalence are the same. This is partly a reflection of the complexities of manufacturing and safety and efficacy controls of biosimilars compared with their small-molecule generic counterparts. ${ }^{1-4}$ Since biologic products are often recombinant protein molecules manufactured in living cells, ${ }^{5}$ manufacturing processes for biologic products are highly complex and require hundreds of specific isolation and purification steps. In practice, it is impossible to produce an identical copy of a biologic product, as changes to the structure of the molecule can occur with changes in the production process. Since a protein can be modified during the process (eg, a side chain may be added, the structure may have changed due to protein misfolding, and so on), different manufacturing processes may lead to structural differences in the final product, which result in differences in efficacy and safety, and may have a negative impact on the immune responses of patients. It should be noted that these issues occur also during the postapproval changes of the innovator's biological products.

In this article, we will focus on scientific factors for assessing biosimilarity and drug interchangeability of follow-on biologics as discussed at the FDA Part 15 Public Hearing on Approval Pathway for Biosimilar and Interchangeability Biological Products held on November 2-3, 2010 in Silver Spring, Maryland. In addition, statistical issues surrounding the assessment of biosimilarity of follow-on biologics including manufacturing, quality control, clinical efficacy, side-effects (safety), and immunogenicity will also be briefly reviewed. Recommendations will be made to address these issues whenever possible.

In the next section, past experiences for the assessment of bioequivalence for the generic approval of conventional drug products are given. Further sections describe fundamental differences and assumptions between conventional drug products and follow-on biologics; briefly, current regulatory requirements for the approval of biosimilars by the European Union (EU) EMA, and the current position of the FDA; statistical considerations for scientific factors for assessing biosimilarity and drug interchangeability of follow-on biologics; and statistical issues on critical attributes of a potential patient's response in follow-on biologics. Brief concluding remarks are given in the last section.

\section{Past experience in bioequivalence}

For an approval of generic drug products, the FDA requires that evidence of average bioequivalence be provided in terms of some pharmacokinetic parameters such as the area under the blood and/or plasma concentration-time curve (AUC) and peak concentration $\left(\mathrm{C}_{\max }\right)$. We claim that a test drug product is bioequivalent to a reference (innovative) drug product if the $90 \%$ confidence interval for the ratio of geometric means (in \%) of the primary PK parameter is totally within the bioequivalence limits of $80 \%$ to $125 \%$. The confidence interval for the ratio of geometric means of the primary PK parameter is obtained based on the log-transformed metrics. In what follows, study designs and statistical methods that are commonly considered in bioequivalence studies are briefly introduced.

\section{Study design}

As indicated in the Federal Register [Vol. 42, No. 5, Sec. 320.26(b) and Sec. 320.27(b), 1977], a bioequivalence study (single-dose or multi-dose) should be crossover in design, unless a parallel or other design is more appropriate for valid scientific reasons. Thus, in practice, a standard two-sequence, two-period (or $2 \times 2$ ) crossover design is often considered for a bioequivalence study. Denote by $\mathrm{T}$ and $\mathrm{R}$ the test product and the reference product, respectively. Thus, a $2 \times 2$ crossover design can be expressed as (TR, RT), where $\mathrm{TR}$ is the first sequence of treatments and RT denotes the second sequence of treatments. Under the (TR, RT) design, qualified subjects who are randomly assigned to sequence 1 (TR) will receive the test product $(\mathrm{T})$ first and then get the reference product $(\mathrm{R})$ after a sufficient length of washout period. Similarly, subjects who are randomly assigned to sequence 2 (RT) will be administered the reference product (R) first and then receive the test product (T) after a sufficient length of washout period.

One of the limitations of the standard $2 \times 2$ crossover design is that it does not provide independent estimates of intra-subject variabilities since each subject will receive the same treatment only once. In the interest of assessing intrasubject variabilities, the following alternative designs for comparing two drug products are often considered:

1. Balaam's design - (TT, RR, RT, TR);

2. Two-sequence, three-period dual design - (TRR, RTT); 
3. Four-sequence, four-period design - (TTRR, RRTT, TRTR, RTTR).

These study schemes are also referred to as higherorder crossover designs. A higher-order crossover design is defined as a design with the number of sequences or the number of periods higher than the number of treatments to be compared.

For assessing population and/or individual bioequivalence, FDA recommended that a replicated design be considered for obtaining independent estimates of intra-subject variabilities, inter-subject variabilities, and variability due to subject-by-drug product interaction. ${ }^{6}$ A commonly considered replicate crossover design is the replicate of a $2 \times 2$ crossover design, which is given by (TRTR, RTRT).

In some cases, an incomplete block design or an extrareference design such as (TRR, RTR) may be considered depending upon the study objectives of the bioavailability/ bioequivalence studies. $^{7}$

\section{Statistical methods}

As indicated earlier, bioequivalence is claimed if the ratio of geometric mean bioavailabilities between test and reference products is (in \%) within the bioequivalence limits of $(80 \%$, $125 \%$ ) with $90 \%$ assurance based on log-transformed data. Along this line, commonly employed statistical methods are the confidence interval approach and the method of interval hypotheses testing. For the confidence interval approach, a $90 \%$ confidence interval for the ratio of means of the primary pharmacokinetic response such as AUC or $\mathrm{C}_{\text {max }}$ is obtained under an analysis of variance model. We claim bioequivalence if the obtained $90 \%$ confidence interval is totally within the bioequivalence limit of $(80 \%, 125 \%)$. For the method of interval hypotheses testing, the interval hypotheses that

\section{$H_{0}$ : Bioinequivalence versus $H_{a}$ : Bioequivalence}

are decomposed into two sets of one-sided hypotheses. The first set of hypotheses verifies that the average bioavailability of the test product is not too low, whereas the second set of hypotheses verifies that average bioavailability of the test product is not too high. Schuirmann's two one-sided tests procedure is commonly employed for the interval hypotheses testing average bioequivalence (ABE) ${ }^{8}$

Other statistical methods such as Westlake's symmetric confidence interval approach, a confidence interval based on Fieller's theorem, Chow and Shao's joint confidence region approach, Bayesian methods, and nonparametric methods (eg, Wilcoxon-Mann-Whitney two one-sided tests procedure, distribution-free confidence interval based on the Hodges-Lehmann estimator, and bootstrap confidence interval) are sometimes considered. Details of these methods can be found in Chow and Liu. ${ }^{9}$

\section{Remarks}

Although the assessment of ABE for generic approval has been in practice for years, it has the following limitations: 1) it focuses only on the population average; 2) it ignores the distribution of the metric; 3) it does not provide independent estimates of intra-subject variability and ignores the subject-by-formulation interaction. Many authors criticize that 1) ABE utilizes a one-size-fits-all criterion which should be adjusted for intra-subject variability and/or therapeutic index of the drug product under investigation, and 2) the assessment of $\mathrm{ABE}$ does not address the question of drug interchangeability and may penalize drug products with lower variability.

As indicated by the regulatory agencies, a generic drug can be used as a substitute for the brand-name drug if it has been shown to be bioequivalent to the brand-name drug. Current regulations do not indicate that two generic copies of the same brand-name drug can be used interchangeably even though they are bioequivalent to the same brand-name drug. Bioequivalence between generic copies of a brandname drug is not required. Thus, one of the controversial issues is whether these approved generic drug products can be exchanged safely. Note that in practice, it is possible that there can be drift: one product is bioequivalent but slightly less available than the innovator product and the other also bioequivalent but slightly more available than the innovator formulation. Consequently, the difference between the two generic products could be large enough that they are not bioequivalent.

\section{Fundamental differences from generics and assumptions for biosimilars Fundamental differences from generics}

In comparison with conventional drug products, the concept for the development of follow-on biologics is very different. Webber defines follow-on (protein) biologics as products that are intended to be sufficiently similar to an approved product to permit the applicant to rely on existing scientific knowledge about safety and efficacy of the approved reference product. ${ }^{10}$ Under this definition, follow-on products are not only intended to be similar to the reference product, but also intended to be interchangeable with the reference 
product. As a number of patents for biologic products are due to expire in the next few years, the subsequent follow-on products have generated considerable interest within the pharmaceutical/biotechnological industry as biosimilar manufacturers strive to obtain part of an already large and rapidly growing market. The potential opportunity for price reductions versus the innovator biologic products remains to be determined, as the advantage of a cheaper price may be outweighed by the potentially increased risk of side-effects from biosimilar molecules that are not exact copies of their innovators. In this article, we shall focus on issues surrounding biosimilars, including manufacturing, quality control, clinical efficacy, side-effects (safety), and immunogenicity. In addition, we will also attempt to address the challenges in regulations to deal with these issues.

\section{Fundamental assumptions}

As indicated by Chow and Liu, bioequivalence studies are performed under the so-called Fundamental Bioequivalence Assumption which constitutes legal basis for regulatory approval of generic drug products. ${ }^{9}$ The Fundamental Bioequivalence Assumption states that:

If two drug products are shown to be bioequivalent, it is assumed that they will reach the same therapeutic effect or they are therapeutically equivalent and hence can be used interchangeably.

Note that this seems to have been often interpreted on the basis that the confidence interval for the ratio (in \%) of the geometric means of $\mathrm{AUC}$ and $\mathrm{C}_{\max }$ is between $80 \%$ and $125 \%$. An alternative would be to show that the tolerance intervals (or a distribution-free model) overlap sufficiently.

To protect the exclusivity of a brand-name drug product, the sponsors of the innovator drug products will make every attempt to prevent generic drug products from being approved by the regulatory agencies such as the FDA. One of the strategies is to challenge the Fundamental Bioequivalence Assumption by filing a citizen petition with scientific/clinical justification. Upon the receipt of a citizen petition, the FDA has a legal obligation to respond within 180 days. It should be noted, however, that the FDA will not suspend the review/approval process of a generic submission of a given brand-name drug even if a citizen petition is under review within the FDA.

In spite of the Fundamental Bioequivalence Assumption, one of the controversial issues is that bioequivalence may not necessarily imply therapeutic equivalence and therapeutic equivalence does not guarantee bioequivalence either. The assessment of average bioequivalence for generic approval has been criticized that it is based on legal/ political considerations rather than scientific arguments. In the past several decades, many sponsors/researchers have made attempts to challenge this assumption without success.

In practice, the verification of the Fundamental Bioequivalence Assumption is often difficult, if not impossible, without the conduct of clinical trials. It should be noted that the Fundamental Bioequivalence Assumption is for drug products with identical active ingredient(s). Whether the Fundamental Bioequivalence Assumption is applicable to drug products with similar but different active ingredient(s) as in the case of follow-on products becomes an interesting but controversial question.

Similar to the Fundamental Bioequivalence Assumption described above, it is suggested a Fundamental Biosimilarity Assumption be developed. The following statement could be considered:

When a follow-on biologic product is claimed to be biosimilar to an innovator product in some well-defined study endpoints, it is assumed that they will reach similar thera-

peutic effect or they are therapeutically equivalent.

Some well-defined study endpoints consist of study endpoints from different functional areas such as certain biological activities, pharmacokinetics/pharmacodynamics (PK/PD), immunogenicity, and the manufacturing process.

\section{Current regulatory requirements EMA}

For approval of biosimilars in Europe, the EMA has issued a new guideline describing general principles for the approval of similar biological medicinal products, or biosimilars. ${ }^{11}$ The guideline is accompanied by six concept papers that outline areas in which the agency intends to provide more targeted guidance. ${ }^{12-20}$ Specifically, the concept papers discuss approval requirements for four classes of human recombinant products containing erythropoietin, human growth hormone, granulocyte-colony stimulating factor, and insulin. The guideline consists of a checklist of documents published to date which are relevant to data requirements for biological pharmaceuticals. It is not clear what specific scientific requirements will be applied to biosimilar applications. In addition, it is not clear how the agency will treat innovator data contained in the reference product dossiers. While the guideline provides 
a useful summary of the biosimilar legislation and previous EU publications, it provides few answers to the issues.

\section{The FDA}

In the United States, current approval pathway for follow-on biologics depends on whether the biologic product is approved under the United States Food, Drug, and Cosmetic Act (US FD\&C) or is licensed under the United States Public Health Service Act (US PHS). For products approved under an NDA (US FD\&C Act), generic versions of the products can be approved under an ANDA, eg, under Section 505(b) (2) of FD\&C Act. For products that are licensed under a Biologics License Applications (BLA) (under the US PHS Act), there exists no abbreviated BLA. As pointed out by Woodcock, ${ }^{21}$ for the assessment of similarity of follow-on biologics, the FDA would consider the following factors: 1) the robustness of the manufacturing process, 2) the degree to which structural similarity could be assessed, 3) the extent to which the mechanism of action was understood, 4) the existence of valid, mechanistically related PD assays, 5) comparative PK, 6) comparative immunogenicity, 7) the amount of clinical data available, and 8) the extent of experience with the original product. ${ }^{22-24}$

A typical example would be the recent regulatory authorization of Omnitrope ${ }^{\circledR}$ (somatropin; Sandoz Biopharmaceuticals, Princeton, NJ), which was approved in 2006 under section 505(b) (2) of the FD\&C Act. Omnitrope was approved based on the following evaluations: 1) physicochemical testing that established a highly similar structure to Genotropin, 2) new nonclinical pharmacology and toxicology data specific to Omnitrope, 3) PK, PD, and comparative bioavailability data, 4) clinical efficacy and safety data from comparative controlled trials and from long-term trials with Omnitrope, 5) vast clinical experience and a wealth of published literature on the clinical effects (safety and effectiveness) of human growth hormone. The approval of Omnitrope is based on an ad-hoc, case-by-case review of the individual application for biosimilarity.

In practice, there is a strong industrial interest and desire for the regulatory agencies to develop review standards and an approval process for biosimilars rather than an ad-hoc, caseby-case review of individual biosimilar applications. Under this consideration, FDA indicated that new guidances are currently under development. These guidances will include 1) a guidance for industry on scientific considerations to demonstrate the safety and effectiveness of follow-on protein products, and 2) a guidance for industry on chemistry, manufacturing, and control issues for follow-on protein products.

\section{Scientific factors for assessing biosimilarity Study design}

As indicated, for the assessment of bioequivalence of drug products, the standard two-sequence, two-period crossover scheme is the design of choice. However, for the assessment of biosimilarity between an innovative product and its follow-on biologics, it is a concern whether a crossover design is feasible especially when the biological drug product has a relatively long half-life. As a result, it is suggested that a parallel-group design should be considered unless there is evidence that: 1) the half-life of the biological product is relatively short, and 2) there is no carry-over effect after a sufficient length of washout. Note that, alternatively, a twostage design combining a crossover scheme with a limited number of subjects and a parallel group design could be considered if the estimation of intra-subject variability is of interest to the investigator. Furthermore, a Bayesian adaptive design may be considered if prior information of the product is available and/or some adaptations are of interest to the investigator.

Since some of the biological products such as therapeutic antibodies or pegylated proteins have a long half-life, equivalence in terms of absorption/bioavailability may not be sufficient. Demonstration of equivalence of clearance and half-life may be required for assessing the risk of difference in elimination rate. As a result, the traditional crossover designs may not be optimal for the evaluation of equivalence between follow-on and innovator biological products. On the other hand, if the well-defined and validated product characteristics are $\mathrm{PK} / \mathrm{PD}$ responses, it is then very important to investigate the ability to extrapolate the equivalence in PK responses to the equivalence in PD as well as in efficacy responses. In order to ensure the internal validity of treatment comparisons, PK, PD, and efficacy responses should be evaluated simultaneously in the same trials. Chow and $\mathrm{Liu}^{25}$ consider Design A illustrated in Figure 1.

Design A is a two-group parallel design in patients for a PK/PD/efficacy bridging study with the disease which the biological product of the innovator is indicated for. After meeting the inclusion and exclusion criteria, patients are randomly divided into two groups. PD/efficacy/safety will be evaluated for the first group of the patients (validation set). Additional PK responses will be assessed for the second group of the patients (training set). A randomization in a 1:1 ratio will be performed separately for each group. The sample size of the second group will be large enough to 


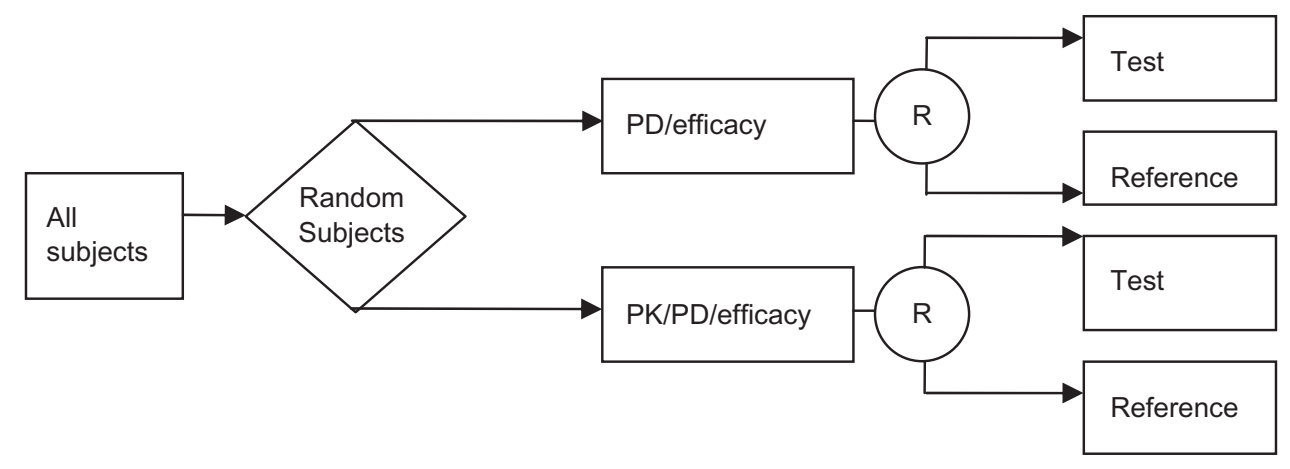

Figure I Design A for a PK/PD/efficacy bridging study.

Abbreviations: PD, pharmacodynamics; PK, pharmacokinetics.

provide sufficient power for the evaluation of bioequivalence/ biosimilarity based on PK responses. Calibration models will be built based on the PK/PD/efficacy responses obtained from the patients in the training set. The PD/efficacy data from the validation set will be used to provide an independent assessment of the ability to extrapolate the equivalence of PK responses to the equivalence in $\mathrm{PD} /$ efficacy responses.

Design A may require quite large sample sizes because of the simultaneous evaluation of the ability to extrapolate the equivalence in PK to the equivalence in PD and in efficacy. One way to resolve this issue is to adopt the design for dose-response trials for the evaluation of the ability to extrapolate of the equivalence in some well-defined product characteristics to the equivalence in efficacy. This design is referred to as Design B (Figure 2). Design B consists of two dose-response trials: one for the biosimilar product and one for the innovator's biological product, each with at least three dose levels with a placebo group. Eligible patients are first randomized into biosimilar or innovator groups. Within each group, patients are randomized again to receive one of the doses for the respective products. Well-defined product characteristics and primary efficacy endpoints are evaluated for all patients at their respective doses. Suppose that a statistically significant relationship as represented by a simple

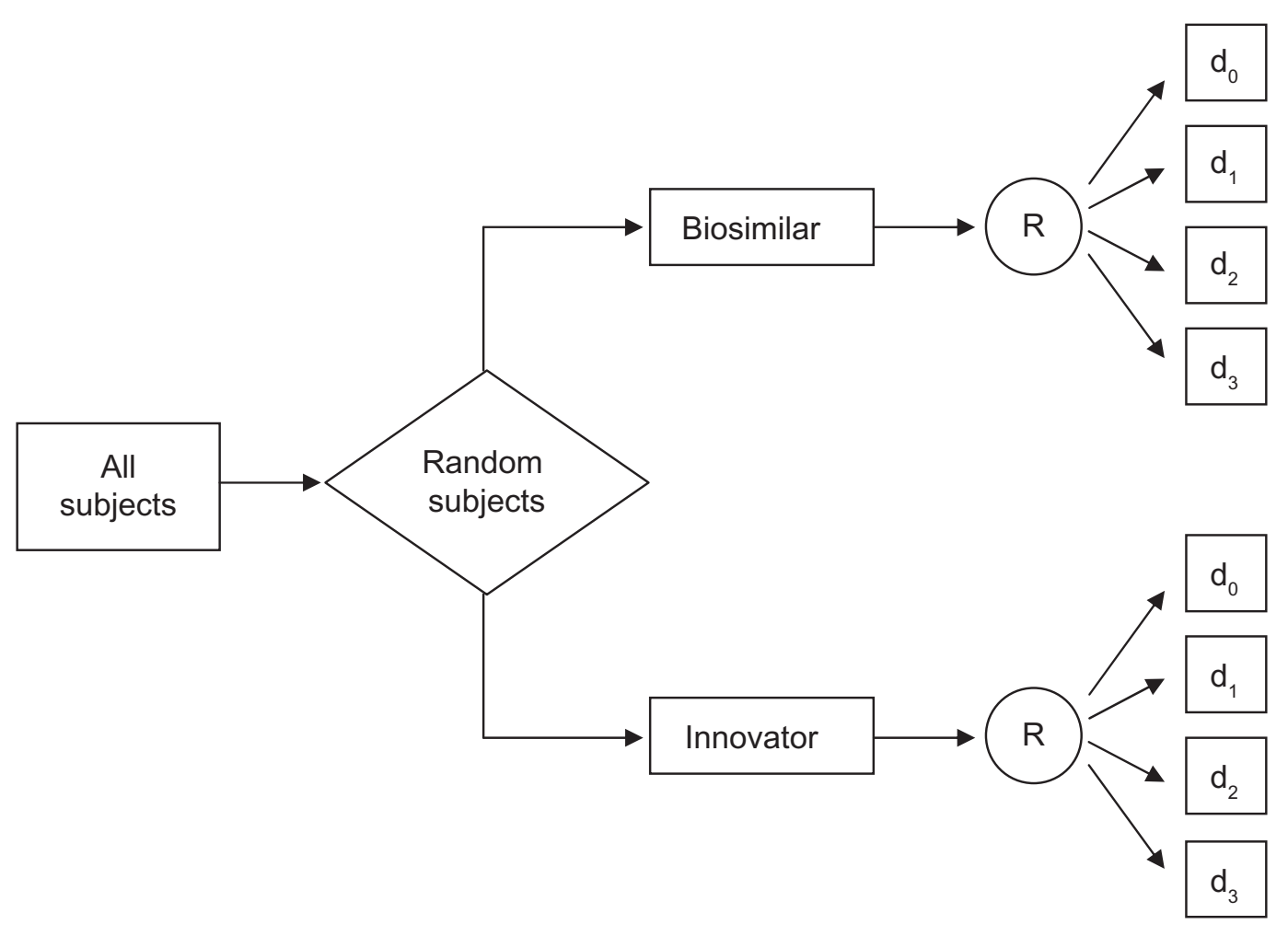

Figure 2 Design B for the evaluation of extrapolation ability. 
linear regression equation can be established, maybe after a suitable transformation, between the well-defined product characteristics and the primary efficacy endpoint through dose levels for the innovator's product. If a similar linear relationship can be also obtained for the biosimilar product, and its corresponding linear regression equation is very close to the one for the innovator's product, then equivalence in efficacy based on the primary efficacy endpoint may be claimed. Because the innovator's product has been approved by the regulatory agencies due to its confirmed efficacy, therefore the objective of Design B is not to establish the efficacy of either biological product but to establish the similar patterns of the relationship between the well-defined product characteristics and primary efficacy endpoint for the two products. As a result, the sample size of Design B can be reduced significantly.

\section{Criteria for biosimilarity}

For the comparison between drug products, some criteria for the assessment of bioequivalence, similarity (eg, the comparison of dissolution profiles), and consistency (eg, comparisons between manufacturing processes) are available in either regulatory guidelines/guidances and/or the literature. These criteria, however, can be classified into either 1) absolute change versus relative change, 2) aggregated versus disaggregated, or 3) moment-based versus probability-based. In this section, different categories of criteria are briefly reviewed.

\section{Absolute change versus relative change}

In clinical research and development, for a given study endpoint, either post-treatment absolute change or post-treatment relative change from a baseline is usually considered for comparisons between treatment groups. A typical example would be the study of weight reduction in an obese patient population. In practice, it is not clear whether a clinically meaningful difference in terms of an absolute change from a baseline can be translated to a clinically meaningful difference in terms of a relative change from the baseline. Sample size calculations based on power analysis in terms of an absolute change from a baseline or a relative change from a baseline could lead to very different results.

Current regulations for the assessment of bioequivalence between drug products in terms of average bioavailability are based on relative change. In other words, we conclude (average) bioequivalence between a test product and a reference product if the $90 \%$ confidence interval for the ratio of geometric means of the primary PK response such as the
AUC between the two drug products is (in percent) totally within $80 \%$ and $125 \%$. Note that regulatory agencies suggest that a log-transformation be performed before data analysis for the assessment of bioequivalence.

\section{Aggregated versus disaggregated criteria}

As indicated by Chow and Liu, ${ }^{9}$ bioequivalence can be assessed by evaluating separately differences in averages, intra-subject variabilities, and the variance due to subjectby-formulation interaction between drug products. Separate criteria for the assessment of differences in averages, intrasubject variabilities, and the variance due to subject-byformulation interaction between drug products are referred to as disaggregated criteria. If the criterion is a single summary measure composed of these individual criteria, it is called an aggregated criterion.

For the assessment of $\mathrm{ABE}$, most regulatory agencies, including the FDA, recommend the use of a disaggregate criterion based on average bioavailability. In other words, bioequivalence is concluded if the average bioavailability of the test formulation is between $80 \%$ and $125 \%$ of the $\mathrm{ABE}$ for the reference formulation, with a certain assurance. Note that $\mathrm{EMA}^{26}$ and $\mathrm{WHO}^{27}$ use the same equivalence criterion of $80 \%$ to $125 \%$ for the log-transformed pharmacokinetic responses such as AUC. However, for $\mathrm{C}_{\max }$, in certain cases, the EMA and WHO allow a wider interval of $75 \%$ to $133 \%$ for the ratio of average bioavailability to address any safety and efficacy concerns for patients being switched between formulations. If a wider interval is used, it must be prespecified in the protocol. More details can be found in Chow and Liu. ${ }^{9}$

On the other hand, for the assessment of population bioequivalence (PBE) and individual bioequivalence (IBE), the following aggregated criteria have been considered. For the assessment of individual bioequivalence, a criterion proposed in the FDA guidance ${ }^{6}$ can be expressed as:

$$
\theta_{I}=\left(\delta^{2}+\sigma_{D}^{2}+\sigma_{W T}^{2}-\sigma_{W R}^{2}\right) / \max \left\{\sigma_{W 0}^{2}, \sigma_{W R}^{2}\right\}
$$

where $\delta=\mu_{T}-\mu_{R}, \sigma_{W T}^{2}, \sigma_{W R}^{2}, \sigma_{D}^{2}$ are the true difference between means, the intra-subject variances of the test product and the reference product, and variance component due to subject-by-formulation interaction, respectively. $\sigma_{W 0}^{2}$ is a scale parameter specified by the regulatory agency. Similarly, the criterion for the assessment of population bioequivalence suggested in the FDA guidance ${ }^{6}$ is given by

$$
\theta_{P}=\left(\delta^{2}+\sigma_{T T}^{2}-\sigma_{T R}^{2}\right) / \max \left\{\sigma_{T 0}^{2}, \sigma_{T R}^{2}\right\},
$$


where $\sigma_{T T}^{2}, \sigma_{T R}^{2}$ are the total variances for the test product and the reference product, respectively, and $\sigma_{T 0}^{2}$ is a scale parameter specified by the regulatory agency.

A typical approach is to construct a one-sided $95 \%$ confidence interval for $\theta_{I}\left(\theta_{P}\right)$ for the assessment of individual (population) bioequivalence. If the one-sided $95 \%$ upper confidence limit is less than the bioequivalence limit of $\theta_{I}\left(\theta_{P}\right)$, then we conclude that the test product is bioequivalent to that of the reference product in terms of individual (population) bioequivalence. More details on individual and population bioequivalence can be found in Chow and Liu. ${ }^{9}$

Note that although individual bioequivalence has been discussed tremendously in the past, it has been dropped by the FDA and is no longer used or considered.

\section{Moment-based criteria versus probability-based criteria}

Schall and Luus proposed moment-based and probability-based measures for the expected discrepancy in pharmacokinetic responses between drug products. ${ }^{28}$ The moment-based measure suggested by Schall and Luus ${ }^{28}$ defines the following expected mean-squared differences:

$$
d\left(Y_{j} ; Y_{j^{\prime}}\right)=\left\{\begin{array}{c}
E\left(Y_{T}-Y_{R}\right)^{2} \text { if } j=T \text { and } j^{\prime}=R \\
E\left(Y_{R}-Y_{R}^{\prime}\right)^{2} \text { if } j=R \text { and } j^{\prime}=R .
\end{array}\right.
$$

For some prespecified positive number $r$, one of probability-based measures for the expected discrepancy is given as: ${ }^{28}$

$$
d\left(Y_{j} ; Y_{j^{\prime}}\right)= \begin{cases}P\left\{\left|Y_{T}-Y_{R}\right|<r\right\} & \text { if } j=T \text { and } j^{\prime}=R \\ P\left\{\left|Y_{R}-Y_{R}^{\prime}\right|<r\right\} & \text { if } j=R \text { and } j^{\prime}=R .\end{cases}
$$

$d\left(Y_{T} ; Y_{R}\right)$ measures the expected discrepancy for some pharmacokinetic metric between the test and reference formulations, and $d\left(Y_{R} ; Y_{R}{ }^{\prime}\right)$ provides the expected discrepancy between the repeated administrations of the reference formulation. The role of $d\left(Y_{R} ; Y_{R}{ }^{\prime}\right)$ in the formulation of bioequivalence criteria is to serve as a control. The rationale is that the reference formulation should be bioequivalent to itself. Therefore, for the moment-based measures, if the test formulation is indeed bioequivalent to the reference formulation, then $d\left(Y_{T} ; Y_{R}\right)$ should be very close to $d\left(Y_{R} ; Y_{R}{ }^{\prime}\right)$. It follows that if the criteria are functions of the difference (or ratio) between $d\left(Y_{T} ; Y_{R}\right)$ and $d\left(Y_{R} ; Y_{R}{ }^{\prime}\right)$, bioequivalence is concluded if they are smaller than some prespecified limit. On the other hand, for probability-based measures, if the test formulation is indeed bioequivalent to the reference formulation, compared with $d\left(Y_{R} ; Y_{R}^{\prime}\right) d\left(Y_{T} ; Y_{R}\right)$, should be relatively large. As a result, bioequivalence is concluded if the criteria based on the probability-based measure is higher than some prespecified limit.

\section{Remarks}

Although several criteria for similarity are available in both regulatory guidelines/guidances and the literature, these criteria are not interchangeable. In other words, one may pass one criterion but fail to pass others. Besides, these criteria do not address the critical questions for assessing follow-on biologics: 1) how similar is similar? and 2) the impact of the level of similarity on drug interchangeability. Based on extensive simulation studies, following the concept for assessment of bioequivalence, Chow et $\mathrm{al}^{29}$ and Hsieh et al ${ }^{30}$ suggest a probability-based criterion on relative change be considered for assessment of follow-on biologics.

Under a valid study design and appropriate criteria for biosimilarity, relevant statistical methods can be derived. For example, for the assessment of bioequivalence/biosimilarity, Schuirmann's two one-sided tests procedure and/or confidence interval approach can be used.

\section{Scientific factors for assessing drug replacement}

For small-molecule drug products, the replacement of one drug product by another can be classified either as drug prescribability or drug switchability. Drug prescribability is defined as the physician's choice for prescribing an appropriate drug product for their new patients between a brand-name drug product and a number of generic drug products that have been shown to be bioequivalent/biosimilar to the brand-name drug product. The underlying assumption of drug prescribability is that the brand-name drug product and its generic copies can be used alternatively in terms of the efficacy and safety of the drug product. Drug prescribability, therefore, is the choice of a drug product for a new patient who has not yet received the drug in any of its forms.

Drug switchability, on the other hand, is related to the switch from a drug product (eg, a brand-name drug product) to an alternative drug product (eg, a generic copy of the brand-name drug product) within the same subject, whose concentration of the drug product has been titrated to a steady, efficacious, and safe level. As a result, drug switchability is considered more critical than drug prescribability for patients who have been on medication for a while. Drug switchability, therefore, is replacement within the same subject. 
Note that in practice, many use the terms interchangeability and switchability synonymously. (Another term used in this context is substitutability). These terms are meant to replace, in a given patient, the administration of one drug product by another. Thus, these usages refer to subjects to whom the drug has already been administered and who are not naïve to it. The recent Canadian document is an example of the widespread usage of these terms. Also noteworthy is the definition of interchangeability in the Biologics Price Competition and Innovation Act of 2009, Section 7002: “(3) The term 'interchangeable' or 'interchangeability', in reference to a biological product that is shown to meet the standards described in subsection $(\mathrm{k})(4)$, means that the biological product may be substituted for the reference product without the intervention of the health care provider who prescribed the reference product".

\section{Population bioequivalence for drug prescribability}

As indicated in Chow and Liu, ${ }^{9} \mathrm{ABE}$ can guarantee neither drug prescribability nor drug switchability. Therefore, it is suggested that the assessment of bioequivalence should take into consideration drug prescribability and drug switchability. To address drug replacement, it is recommended that PBE and IBE be considered for testing drug prescribability and drug switchability, respectively. More specifically, the FDA has recommended that PBE be applied to new formulations, additional strengths, or new dosage forms in NDAs, while IBE should be considered for ANDA or AADA (abbreviated antibiotic drug application) for generic drugs.

To address drug prescribability, the FDA proposed the following aggregated, scaled, moment-based, one-sided criterion:

$$
\begin{aligned}
& \text { Population } \\
& \text { bioequivalence } \\
& \text { criterion (PBC) }
\end{aligned}=\frac{\left(\mu_{T}-\mu_{R}\right)^{2}+\left(\sigma_{T T}^{2}-\sigma_{T R}^{2}\right)}{\max \left(\sigma_{T R}^{2}, \sigma_{T 0}^{2}\right)} \leq \theta_{P},
$$

where $\mu_{T}$ and $\mu_{R}$ are the logarithmic means of the test drug product and the reference drug product, respectively, $\sigma_{T T}^{2}$ and $\sigma_{T R}^{2}$ are the total variances of the test drug product and the reference drug product, respectively, $\sigma_{T 0}^{2}$ is a constant that can be adjusted to control the probability of passing PBE, and $\theta_{P}$ is the bioequivalence limit for PBE. The numerator on the left-hand side of the criterion is the sum of the squared difference between the population averages and the difference of total variances between the test and reference drug products which measure the similarity for the marginal population distribution between the test and reference drug products.
The denominator on the left-hand side of the criterion is a scaling factor that depends upon the variability of the drug class of the reference drug product. The FDA guidance suggests that $\theta_{P}$ be chosen as

$$
\theta_{P}=\frac{(\log 1.25)^{2}+\varepsilon_{P}}{\sigma_{T 0}^{2}}
$$

where $\varepsilon_{P}$ is guided by taking into account of adding the variability term $\sigma_{T T}^{2}-\sigma_{T R}^{2}$ to the $\mathrm{ABE}$ criterion. As suggested by the FDA guidance, it may be appropriate that $\varepsilon_{P}$ chosen to be 0.02 . For the determination of $\sigma_{T 0}^{2}$, the guidance suggests the use of so-called population difference ratio (PDR), which is defined as

$$
\begin{aligned}
P D R & =\left[\frac{E(T-R)^{2}}{E\left(R-R^{\prime}\right)^{2}}\right]^{1 / 2} \\
& =\left[\frac{\left(\mu_{\mathrm{T}}-\mu_{R}\right)^{2}+\sigma_{T T}^{2}+\sigma_{T R}^{2}}{2 \sigma_{T R}^{2}}\right]^{1 / 2} \\
& =\left[\frac{P B C}{2}+1\right]^{1 / 2} .
\end{aligned}
$$

Therefore, assuming that the maximum allowable PDR is 1.25 , substitution of $(\log 1.25)^{2} / \sigma_{T 0}^{2}$ for PBC without adjustment of the variance term approximately yield $\sigma_{T 0}=0.2$.

\section{Individual bioequivalence for drug switchability}

Similarly, to address drug switchability, the FDA recommended the following aggregated, scaled, moment-based, one-sided criterion:

$$
I B C=\frac{\left(\mu_{T}-\mu_{R}\right)^{2}+\sigma_{D}^{2}+\left(\sigma_{W T}^{2}-\sigma_{W R}^{2}\right)}{\max \left(\sigma_{W R}^{2}, \sigma_{W 0}^{2}\right)} \leq \theta_{I},
$$

where $\sigma_{W T}^{2}$ and $\sigma_{W R}^{2}$ are the within-subject variances of the test drug product and the reference drug product, respectively, $\sigma_{D}^{2}$ is the variance component due to subject-by-drug interaction, $\sigma_{W 0}^{2}$ is a constant that can be adjusted to control the probability of passing IBE, and $\theta_{I}$ is the bioequivalence limit for IBE. The FDA guidance suggests that $\theta_{I}$ be chosen as

$$
\theta_{I}=\frac{(\log 1.25)^{2}+\varepsilon_{I}}{\sigma_{W 0}^{2}},
$$

where $\varepsilon_{I}$ is the variance allowance factor, which can be adjusted for sample size control. Note that the FDA guidance suggests $\varepsilon_{I}=0.05$. The determination of $\sigma_{W 0}^{2}$ can be similarly obtained using the individual difference ratio as suggested in 
the FDA guidance. It should be noted that although the FDA guidance recommends $\sigma_{W O}=0.2$, FDA uses (in a different context) $\sigma_{W O}=0.25$.

\section{Alternating and switching for follow-on biologics}

Although similar ideas for assessing drug interchangeability for conventional drug products can be applied to follow-on biologics if independent estimates of intra-subject variabilities, inter-subject variabilities, and the variability due to subject-by-drug interaction can be obtained under a valid study design, the FDA has little perception of drug interchangeability for follow-on biologics. The concept of drug interchangeability of follow-on biologics includes switching and alternating between an innovative biologic product (R) and its follow-on biologics (T).

The concept of switching is referred to as not only the switch from $\mathrm{R}$ to $\mathrm{T}$ or $\mathrm{T}$ to $\mathrm{R}$ (in the narrow sense of switchability), but also $\mathrm{T}$ to $\mathrm{T}$ and $\mathrm{R}$ to $\mathrm{R}$ (in the broader sense of switchability). As a result, biosimilarity for 1) $\mathrm{R}$ to $\mathrm{T}$, 2) $T$ to $R, 3$ ) $T$ to $T$, and 4) $R$ to $R$ needs to be assessed for addressing the concept of switching based on some biosimilarity criterion under a valid study design. In order to assess biosimilarity for 1) $\mathrm{R}$ to $\mathrm{T}, 2$ ) $\mathrm{T}$ to $\mathrm{R}, 3$ ) $\mathrm{T}$ to $\mathrm{T}$, and 4) $\mathrm{R}$ to R, Balaam's $4 \times 2$ crossover design, ie, (TT, RR, TR, RT) may be useful.

On the other hand, the concept of alternating is referred to as 1) the switch from $T$ to $R$ and then switch back to $T$, and 2) the switch from R to T and then switch back to R. Thus, the difference between "the switch from $\mathrm{T}$ to $\mathrm{R}$ " and "the switch from R to T" needs to be assessed for addressing the concept of alternating for "the switch from $\mathrm{T}$ to $\mathrm{R}$ and then switch back to T". Similarly, the difference between "the switch from $\mathrm{R}$ to $\mathrm{T}$ " and "the switch from $\mathrm{T}$ to $\mathrm{R}$ " has to be assessed for addressing the concept of alternating for "the switch from $\mathrm{R}$ to $\mathrm{T}$ and then switch back to $\mathrm{R}$ ". For addressing the concept of alternating as described above, a $2 \times 3$ dual design, ie, (TRT, RTR) may be useful.

For addressing both concepts of switching and alternating for drug interchangeability of follow-on biologics, a modified Balaam's crossover design, ie, (TT, RR, TRT, RTR) is recommended.

\section{Remarks}

As indicated in Chen et al, ${ }^{31}$ individual bioequivalence for drug products is to address drug switchability. The FDA indicated that variability due to the subject-by-formulation interaction could be used as an indicator of drug switchability and suggested that a value of 0.15 for the estimation of the standard deviation due to the subject-by-formulation may be considered to be important. ${ }^{32}$ However, positive bias observed for the estimation of the variability due to the subject-byformulation interaction was observed in a numerical study by Endrenyi et al, ${ }^{33}$ which showed about a quarter to onethird of estimates $>0.15$. Thus, the estimate of $\sigma_{D}$ does not result from the true existence of the subject-by-formulation interaction rather than the large intra-subject variability of the reference formulation. As a result, an estimate of $\sigma_{\mathrm{D}} / \sigma_{\mathrm{WR}}$ is suggested as an alternative indicator for addressing drug switchability.

For addressing the concept of switching and/or alternating for drug interchangeability of follow-on biologics, Chow et $\mathrm{al}^{34}$ suggested the use of biosimilar index, which is derived based on reproducibility probability ${ }^{35}$ for the assessment of biosimilarity. As indicated by Chow et al, ${ }^{34}$ the use of biosimilar index has the advantages that 1) it follows the well-established criterion for the assessment of bioequivalence, which has been used for decades, 2) the probability of reproducibility will reflect the sensitivity of heterogeneity in variation, 3) it can address not only the question that "how similar is similar", but also switching and/or alternating for drug interchangeability.

\section{Current statistical issues}

In addition to the scientific factors related to the assessment of biosimilarity and drug interchangeability of follow-on biologics, several statistical issues regarding critical attributes of a potential patient's response in follow-on biologics were also posted at the FDA Public Hearing on Approval Pathway for Biosimilar and Interchangeability Biological Products, which are briefly described below.

\section{Biosimilarity in biological activity}

Pharmacological or biological activity is an expression describing the beneficial or adverse effects of a drug on living matter. When the drug is a complex chemical mixture, this activity is exerted by the substance's active ingredient or pharmacophore but can be modified by the other constituents. A crucial component of biological activity is a substance's toxicity. Activity is generally dosage-dependent, and it is not uncommon to have effects ranging from beneficial to adverse for one substance when going from low to high doses. Activity depends critically on the fulfillment of the absorption, distribution, metabolism, and excretion criteria.

Note that the EU Pharmaceutical Review legislation published on April 30, 2004 amended the EU community 
code on medicinal products to provide for the approval of biosimilars based on less preclinical and clinical data than had been required for the original reference product. The complexity of the protein and knowledge of its structurefunction relationships determine the type of information needed to establish similarity.

\section{Similarity in size and structure}

In practice, sponsors perform various in vitro tests such as the assessments of the primary amino acid sequence, charges and hydrophobic properties to compare the structural aspects of biosimilars with their originator molecules. However, it is a concern whether in vitro tests can be predictive of biological activity in vivo due to the fact that there may be significant differences in biological activity despite similarities in size and structure. Besides, it is difficult to assess biological activity adequately as few animal models are able to provide data that can be extrapolated for an accurate and reliable prediction of biological activity in humans. Thus, controlled clinical trials remain the most reliable means of demonstrating similarity between a biosimilar molecule and the originator product.

\section{The problem of immunogenicity}

Since all biologic products are biologically active molecules derived from living cells and have the potential to evoke an immune response, immunogenicity is probably the most critical safety concern for the assessment of biosimilarity of follow-on biologics. The commonly seen possible causes of immunogenicity include, but are not limited to: 1) sequence differences between a therapeutic protein and endogenous proteins, 2) nonhuman sequences or epitopes, 3) structural alterations, 4) storage conditions, 5) purification during the manufacturing process, 6) formulation (eg, surfactants), 7) route, dose and frequency of administration, 8) patient status such as genetic background. Thus, the following questions should be asked when assessing biosimilarity between biological products: 1) What is the immunogenic potential of the therapeutic protein? 2) What is the impact of the generating antibodies to the self protein? 3) What is the impact of immunogenicity on preclinical toxicity (eg, pharmacokinetic levels and dose limiting toxicity)? 4) What is the impact of immunogenicity of the therapeutic protein on safety? 5) What are the risk evaluation and mitigation strategy processes required by a regulatory agency such as FDA?

The immune responses to biologic products can lead to: 1) anaphylaxis, 2) injection site reactions, 3) flu-like syndromes, and 4) allergic responses. Note that one of the most serious adverse events occurs when neutralizing antibodies cross react with endogenous proteins that have a unique physiological role. The risk of immunogenicity can be reduced through stringent testing of the products during its development. It should be noted, however, that immunogenicity in animals does not predict immunogenicity in clinical trials, and also analytical techniques may not detect changes that may affect immunogenicity. Therefore, the immunogenicity of a biological product depends heavily upon the attributes of product quality such as the physical, structural, and functional properties of the active pharmaceutical ingredients; as well as excipients, container closure, and delivery system. It turns out that similarity of the acceptable ranges of these quality attributes is crucial to the evaluation of similarity between biosimilars and innovator's products.

\section{Manufacturing process}

Unlike small-molecule drug products, biological products are made of living cells. Thus, manufacturing of biologic products is a very complicated process, which involves the steps of 1) cell expansion, 2) cell production (in bioreactors), 3) recovery (through filtration or centrifugation), 4) purification (through chromatography), and 5) formulation. A small discrepancy at each step (eg, purification) could lead to a significant difference in the final product, which might cause a drastic change in clinical outcomes. Thus, process control and validation play an important role for the success of the manufacturing of biological products. In addition, since at each step (eg, purification), different methods may be used at different biological manufacturing processes (within the same company or at different biotech companies), tests for consistency are necessarily performed. Note that at the step of purification, the following chromatography media or resins are commonly considered: 1) gel filtration, 2) ion exchange, 3) hydrophobic interaction, 4) reversed phase normal phase, and 5) affinity. Thus, at each step of the manufacturing process, primary performance characteristics should be identified, controlled, and tested for consistency of process control and validation.

\section{Concluding remarks}

Current methods for the assessment of bioequivalence for drug products with identical active ingredients are not applicable to follow-on biologics due to fundamental differences. The assessment of biosimilarity between follow-on biologics and an innovator product in terms of surrogate endpoints (eg, PK parameters and/or PD responses) or biomarkers (eg, genomic markers) requires the establishment of the 
Fundamental Biosimilarity Assumption in order to bridge the surrogate endpoints and/or biomarker data to clinical safety and efficacy.

Unlike conventional drug products, follow-on biologics can be very sensitive to small changes in variation during the manufacturing process, which have been shown to have an impact on the clinical outcome. Thus, it is a concern whether current criteria and regulatory requirements for the assessment of bioequivalence for drugs with small molecules can be applied also to the assessment of biosimilarity of follow-on biologics. It is suggested that current, existing criteria for the evaluation of bioequivalence, similarity, and biosimilarity be scientifically/statistically evaluated in order to choose the most appropriate approach for assessing biosimilarity of follow-on biologics. It is recommended that the selected biosimilarity criteria should be able to address 1) sensitivity due to small variations in both location (bias) and scale (variability) parameters, and 2) the degree of similarity, which can reflect the assurance for drug interchangeability.

Under the Fundamental Biosimilarity Assumption and the selected biosimilarity criteria, it is also recommended that appropriate statistical methods (eg, comparing distributions and the development of biosimilarity index) be developed under valid study designs (eg, Design A and Design B described earlier) for achieving the study objectives (eg, the establishment of biosimilarity at specific domains or drug interchangeability) with a desired statistical inference (eg, power or confidence interval). To ensure the success of studies conducted for the assessment of biosimilarity of follow-on biologics, regulatory guidelines/guidances need to be developed. Product-specific guidelines/guidances published by the EMA have been criticized for not having standards. Although product-specific guidelines/guidances do not help to establish standards for the assessment of biosimilarity of follow-on biologics, they do provide the opportunity for accumulating valuable experience/information for establishing standards in the future. Thus, several numerical studies are recommended including simulations, meta-analysis, and/or sensitivity analysis, in order to 1) provide a better understanding of these product-specific guidelines/guidances, and 2) check the validity of the established Fundamental Biosimilarity Assumption, which is the legal basis for assessing biosimilarity of follow-on biologics.

Since there are many critical attributes of a potential patient's response in follow-on biologics, for a given critical attribute, valid statistical methods are necessary to be developed under a valid study design and a given set of criteria for similarity, as described in the previous section. Several areas can be identified for developing appropriate statistical methodologies for the assessment of biosimilarity of follow-on biologics. These areas include, but are not limited to:

Criteria for biosimilarity (in terms of average, variability, or distribution).

To address the question of "how similar is similar?", we suggest establishing criteria for biosimilarity in terms of average, variability, and/or distribution.

\section{Criteria for interchangeability}

In practice, it is recognized that drug interchangeability is related to the variability due to subject-by-drug product interaction. However, it is not clear whether a criterion for interchangeability should be based on the variability due to subject-by-drug product interaction or the variability due to subject-by-drug product interaction adjusted for intra-subject variability of the reference drug.

\section{Bridging studies for assessing biosimilarity}

As most biosimilar studies are conducted using a parallel design rather than a replicated crossover design, independent estimates of variance components such as the intra-subject and the variability due to subject-by-drug product interaction are not possible. In this case, bridging studies may be considered.

\section{The use of biosimilar index}

In addition to classical $F$-type test statistics for the assessment of variability, the use of a biosimilar index based on reproducibility probability may be useful.

\section{Comparability in biologic activities}

As indicated by the FDA, testing for the comparability in biologic activities is essential for the assessment of biosimilarity of follow-on biologics. Valid statistical methods are necessary to develop with respect to study design, endpoints, and criteria employed.

\section{Assessment of immunogenicity}

As indicated by the FDA, the assessment of immunogenicity is important for the assessment of biosimilarity of follow-on biologics. Appropriate statistical methods should be developed according to study endpoints and criteria employed.

\section{Consistency in manufacturing processes}

Since a small change in manufacturing process could have a huge impact on the clinical outcome of follow-on 
biologics, tests for consistency in manufacturing processes are very critical in assessment of biosimilarity of follow-on biologics.

\section{Stability testing (multiple labs, multiple lots)}

Since biological products are very sensitive to environmental factors such as light and temperature, we suggest that stability testing be conducted under study designs that are able to account for these environment factors following both International Conference on Harmonization and FDA guidelines for determination of shelf-life.

\section{Sequential testing procedures}

Due to the complexity of the manufacturing process of biological products, sequential testing procedures for statistical quality control may be useful to ensure biosimilarity of follow-on biologics.

\section{Multiple testing procedures}

Since the assessment of biosimilarity of follow-on biologics consists of different domains such as biologic activities, $\mathrm{PK} / \mathrm{PD}$, immunogenicity, and clinical response, we suggest multiple testing procedures to be considered for assessment of globally biosimilarity.

\section{Biomarker for assessing biosimilarity}

Assessing biosimilarity using a surrogate endpoint or biomarker such as genomic data. In addition to PK/PD, biomarkers such as genomic data could serve as surrogate endpoints for the assessment of biosimilarity of follow-on biologics if they are predictive of clinical responses.

\section{Acknowledgments}

This work was supported in part by Amgen, Inc. The authors would like to thank several Amgen colleagues such as Steve Snapinn and Andrew Fox for their constructive discussions and comments that have led to significant improvement of the article.

\section{Disclosure}

The authors report no conflicts of interest.

\section{References}

1. Schellekens H. How similar do 'biosimilar' need to be? Nat Biotechnol. 2004;22:1357-1359.

2. Chirino AJ, Mire-Sluis A. Characterizing biological products and assessing comparability following manufacturing changes. Nat Biotechnol. 2004;22:1383-1391.
3. Crommelin D, Bermejo T, Bissig M, et al. Biosimilars, generic versions of the first generation of therapeutic proteins: do they exist? Contrib Nephrol. 2005;149:287-294.

4. Roger SD, Mikhail A. Biosimilars: opportunity or cause for concern? J Pharm Sci. 2007;10:405-410.

5. Kozlowski S. FDA policy on follow on biologics. Presented at Biosimilars 2007, George Washington University, Washington DC, USA; 2007.

6. Guidance on Statistical Approaches to Establishing Bioequivalence. Rockville, Maryland, USA: Center for Drug Evaluation and Research, the US Food and Drug Administration (FDA); 2001.

7. Chow SC, Shao J, Wang H. Individual bioequivalence testing under $2 \times 3$ crossover designs. Stat Med. 2002;21:629-648.

8. Schuirmann DJ. A comparison of the two one-sided tests procedure and the power approach for assessing the equivalence of average bioavailability. J Pharmacokinet Biopharm. 1987;15:657-680.

9. Chow SC, Liu JP. Design and Analysis of Bioavailability and Bioequivalence Studies. 3rd ed. New York: New York Chapman Hall/CRC Press, Taylor and Francis; 2008.

10. Webber KO. Biosimilars: Are we there yet? Presented at Biosimilars 2007, George Washington University, Washington DC, USA; 2007.

11. EMEA, Draft guideline on similar biological medicinal products containing monoclonal antibodies. EMA/CHMP/BMWP/403543/2010. London, United Kingdom: The European Medicines Agency Evaluation of Medicines for Human Use; 2010.

12. EMEA, Note for Guidance on Comparability of Medicinal Products Containing Biotechnology-derived Proteins as Drug Substance - Non Clinical and Clinical Issues. EMEA/CHMP/3097/02. London, United Kingdom: The European Medicines Agency Evaluation of Medicines for Human Use; 2003.

13. EMEA, Guideline on Comparability of Medicinal Products Containing Biotechnology- derived Proteins as Drug Substance - Quality Issues. London, EMEA/CHMP/ BWP/3207/00/Rev 1. United Kingdom: The European Medicines Agency Evaluation of Medicines for Human Use; 2003.

14. EMEA, Guideline on Similar Biological Medicinal Products. EMEA/ CHMP/437/04. London, United Kingdom: The European Medicines Agency Evaluation of Medicines for Human Use; 2005.

15. EMEA, Draft Guideline on Similar Biological Medicinal Products Containing Biotechnology- derived Proteins as Drug Substance: Quality Issues. EMEA/CHMP/49348/05. London, United Kingdom: The European Medicines Agency Evaluation of Medicines for Human Use; 2005.

16. EMEA, Draft Annex Guideline on Similar Biological Medicinal Products Containing Biotechnology-derived Proteins as Drug Substance - Non Clinical and Clinical Issues - Guidance on Biosimilar Medicinal Products containing Recombinant Erythropoietins. EMEA/ CHMP/94526/05. London, United Kingdom: The European Medicines Agency Evaluation of Medicines for Human Use; 2005.

17. EMEA, Draft Annex Guideline on Similar Biological Medicinal Products Containing Biotechnology-derived Proteins as Drug Substance Non Clinical and Clinical Issues - Guidance on Biosimilar Medicinal Products containing Recombinant Granulocyte-Colony Stimulating Factor. EMEA/CHMP/31329/05. London, United Kingdom: The European Medicines Agency Evaluation of Medicines for Human Use; 2005.

18. EMEA, Draft Annex Guideline on Similar Biological Medicinal Products Containing Biotechnology-derived Proteins as Drug Substance Non Clinical and Clinical Issues - Guidance on Biosimilar Medicinal Products containing Somatropin. EMEA/CHMP/94528/05. London, United Kingdom: The European Medicines Agency Evaluation of Medicines for Human Use; 2005.

19. EMEA, Draft Annex Guideline on Similar Biological Medicinal Products Containing Biotechnology-derived Proteins as Drug Substance - Non Clinical and Clinical Issues - Guidance on Biosimilar Medicinal Products containing Recombinant Human Insulin. EMEA/ CHMP/32775/05. London, United Kingdom: The European Medicines Agency Evaluation of Medicines for Human Use; 2005. 
20. EMEA, Guideline on the Clinical Investigating of the Pharmacokinetics of Therapeutic proteins. EMEA/CHMP/89249/04. London, United Kingdom: The European Medicines Agency Evaluation of Medicines for Human Use; 2005.

21. Woodcock J. The FDA's assessment of follow-on protein products: A historical perspective. Nat Rev Drug Discov. 2007;6:437-442.

22. ICH Q5C Guideline on Quality of Biotechnological Products: Stability Testing of Biotechnological/Biological Products. Rockville, Maryland, USA: Center for Drug Evaluation and Research, Center for Biologics Evaluation and Research, the US Food and Drug Administration; 1996.

23. ICH Q6B Guideline on Test Procedures and Acceptance Criteria for Biotechnological/Biological Products. Rockville, Maryland, USA: Center for Drug Evaluation and Research, Center for Biologics Evaluation and Research, the US Food and Drug Administration; 1999.

24. Q5E Guideline on Comparability of Biotechnological/Biological Products Subject to Changes in Their Manufacturing Process. Rockville, Maryland, USA: Center for Drug Evaluation and Research, Center for Biologics Evaluation and Research, the US Food and Drug Administration; 2005.

25. Chow SC, Liu JP. Statistical assessment of biosimilar products. J Biopharm Stat. 2010;20:10-30.

26. EMEA, Note for Guidance on the Investigation of Bioavailability and Bioequivalence. EMEA/EWP/QWP/1401/98. London, United Kingdom: The European Medicines Agency Evaluation of Medicines for Human Use; 2001.
27. World Health Organization Draft Revision on Multisource (Generic) Pharmaceutical Products: Guidelines on Registration Requirements to Establish Interchangeability. WHO: Geneva, Switzerland; 2005.

28. Schall R, Luus HG. On population and individual bioequivalence. Stat Med. 1993;12:1109-1124.

29. Chow SC, Hsieh TC, Chi E, Yang J. A comparison of moment-based and probability-based criteria for assessment of follow-on biologics. J Biopharm Stat. 2010;20:31-45.

30. Hsieh TC, Chow SC, Liu JP, Hsiao CF, Chi E. Statistical test for evaluation of biosimilarity of follow-on biologics. J Biopharm Stat. 2010;20: 75-89.

31. Chen ML, Patnaik R, Hauck WW, Schuirmann DF, Hyslop T, Williams R. An individual bioequivalence criterion - regulatory considerations. Stat Med. 2000;19:2821-2842.

32. Patnaik RM, Lesko LJ, Chen ML, Williams RL. Individual bioequivalence - new concepts in the assessment of bioequivalence metrics. Clin Pharmacokinet. 1997;33:1-6.

33. Endrenyi L, Taback N, Tothfalusi L. Properties of the estimated variance component for subject-by-formulation interaction in studies of individual bioequivalence. Stat Med. 2000;19:2867-2878.

34. Chow SC, Endrenyi L, Lachenbruch PA. Assessing biosimilarity of follow-on biologics. Scientific Statistical Advisory Board, Amgen, Inc. Thousand Oaks, California. Unpublished white paper; 2010.

35. Shao J, Chow SC. Reproducibility probability in clinical trials. Stat Med. 2002;21:7727-1742.
Biosimilars

\section{Publish your work in this journal}

Biosimilars is an international, peer-reviewed, open access journal focusing on the manufacture, development and medicinal use of biopharmaceutical compounds considered similar to an innovator agent. Specific topics covered in the journal include: Regulatory issues and pathways; manufacturing processes; chemical composition and

\section{Dovepress}

structure; quality and purity; patent issues; bioequivalence and interchangeability; clinical efficacy data; patient perspectives. The manuscript management system is completely online and includes a very quick and fair peer-review system. Visit http://www.dovepress.com/ testimonials.php to read real quotes from published authors. 